Correspondence: Mariya Lorke, Cologne Center for Ethics, Rights, Economics, and Social Sciences of Health (CERES), University of Cologne, Albertus-Magnus-Platz, 50931 Cologne, Germany.

Tel.: +49.0221.470.89126; Fax: +49 (0)221 470-89101.

E-Mail: mariya.lorke@uni-koeln.de

Key words: Health literacy; risk; narrative interviews; reflexive grounded theory.

Acknowledgements: We are obliged to our participants for their time and interest in this study and very thankful for their openness in the research process. This project was conducted at the Cologne Center for Ethics, Rights, Economics, and Social Sciences of Health (CERES) under the leadership of Principal Investigator Christiane Woopen. Furthermore, we are grateful to Rita Schmutzler for her valuable input in the process of defining the inclusion and exclusion criteria and Julia Dick and Leonie Born for making an extremely effective recruiting process possible.

Contributions: ML made a substantial contribution to the conception and design of the study, the analysis and the interpretation of data for the work, and the theoretical embedding of the findings; the author also drafted the article. LH made a substantial contribution to the conception, design, and conduct of the study, and revised the work critically for important intellectual content. KR made a substantial contribution to the conception and design of the work and the recruiting process; the author also revised the work critically for important medical and intellectual content. CW made a substantial contribution to the conception and design of the work and revised the work critically for important intellectual content. SJ made a substantial contribution to the conception, design, and conduct of the study, supervised the research process, and revised the work critically for important intellectual content. All authors gave approval on the final version of the manuscript and agree to be accountable for all aspects of the work.

Conflict of interest: The authors declare that there is no conflict of interest.

Funding: This research was funded by the Robert Bosch Foundation (grant number 11.5.A402.0002.0).

Availability of data and materials: All data generated or analyzed for the purposes of this article are included in this publication.

Ethics approval and consent to participate: Ethics approval was obtained in March 2018, (registration number 18-014) by the ethics committee of the Medical Faculty of the University of Cologne. The study is conformed with the Helsinki Declaration of 1964, as revised in 2013, concerning human and animal rights. All patients participating in this study signed a written informed consent form for participating in this study.

Informed consent: Informed consent is covered by the section on data protection regulations included in the ethics approval.

Received for publication: 28 January 2021.

Accepted for publication: 30 May 2021.

This work is licensed under a Creative Commons Attribution NonCommercial 4.0 License (CC BY-NC 4.0).

${ }^{\circ}$ Copyright: the Author(s), 2021

Licensee PAGEPress, Italy

Qualitative Research in Medicine \& Healthcare 2021; 5:9647

doi:10.4081/qrmh.2021.9647

\section{The ticking time-bomb. Health literacy in the context of genetic risk prediction in familial breast-ovarian cancer; A qualitative study}

\author{
Mariya Lorke, ${ }^{1}$ Laura Harzheim, ${ }^{1}$ Kerstin Rhiem, ${ }^{2}$ \\ Christiane Woopen, ${ }^{1,3}$ Saskia Jünger ${ }^{1,4}$
}

${ }^{1}$ Cologne Center for Ethics, Rights, Economics, and Social Sciences of Health (CERES), University of Cologne and University Hospital of Cologne, Cologne; ${ }^{2}$ Center for Hereditary Breast and Ovarian Cancer, University Hospital Cologne, Cologne; ${ }^{3}$ Research Unit Ethics, Institute for the History of Medicine and Medical Ethics, Faculty of Medicine, University of Cologne and University Hospital of Cologne, Cologne; ${ }^{4}$ Department of Community Health, University of Applied Health Sciences Bochum, Bochum, Germany

\begin{abstract}
Personalised methods of predicting breast and ovarian cancer risk through genetic testing increasingly demand a person's understanding and critical appraisal of risk-related information, as well as decision-making and acting upon disclosure of a positive test result. The current study aims at understanding health literacy (HL) among persons at risk of developing familial breast-ovarian cancer (FBOC) from a bottom-up perspective-incorporating their viewpoints into the research process. Its qualitative design integrates an ethnographic-narrative approach and findings from 10 narrative interviews with women who have undergone genetic testing, analysed by using reflexive grounded theory. The collected data reveal the entanglement of the women's perceptions concerning the risk of getting ill, their identity, and their strategies of managing health. The analysis of this interplay provides an empirical basis for approaching HL in its communicative dimension, considering individuals' understandings of health and illness, and emphasizing the role of critical HL.
\end{abstract}

\section{Introduction and aim of the study}

Breast and ovarian cancer belong to the most common types of cancer among women worldwide. Five to ten percent of all breast and ovarian cancer patients develop the disease due to genetic predisposition. In 30 out of 100 cases of patients with breast or ovarian cancer in Germany, a familial accumulation or an early onset of the disease can be observed. ${ }^{1}$ Predictive genetic testing became a widespread instrument of determining individuals' hereditary susceptibility for familial breast-ovarian cancer (FBOC), and the increasing research in this field allows for more differentiated and individualised knowledge of various genetic and non-genetic risk factors. ${ }^{2}$ 
The German Consortium of Familial Breast and Ovarian Cancer (GC-HBOC), for example, established a multigene panel (TruRisk $\left.{ }^{\circledR}\right)$ for the analysis of risk genes, which currently comprises the core genes $A T M, B A R D 1, B R C A 1$, BRCA2, CDH1, CHEK2, BRIP1, PALB2, RAD51C, $R A D 51 D$ and TP53, as well as other genes still being object of current research that needs further validation.

Apart from specific biographical reasons and motivations that may be crucial for each individual case, most women decide to undergo a genetic test after having developed breast or ovarian cancer due to familial predisposition (based on individual assessment or gynaecologist recommendation) or positive test results of relatives. The increasing opportunities of risk prediction confront individuals with the challenge to find and understand relevant information and to critically appraise it, in order to make a decision concerning genetic testing and preventive interventions. We know a lot about the psychological effects of genetic testing on individuals' identity ${ }^{\mathrm{A}}$ and everyday life, ${ }^{4,5}$ the experiences of affected individuals and their families, ${ }^{6,7}$ individuals' risk perceptions ${ }^{\mathrm{B}}$ in the process of decision-making in terms of prevention, ${ }^{11}$ and the issue of genetic responsibility. ${ }^{12}$ But what do we know about health literacy (HL) among persons at risk of developing FBOC, and what impact does the perception of risk have on individuals' understanding of health and illness and/or vice versa?

Most generally speaking, HL refers to knowledge and skills related to one's health; depending on the respective definition or perspective, this can imply different cognitive, interpersonal, and social skills, and entail a more functional connotation (in terms of basic reading, writing, and literacy skills), a communicative level, and a critical dimension (in terms of appraisal of information or even a

A We define identity here as social identity, ${ }^{3}$ illuminating the human world as selfhood which depends on both an individual's physical body and his/her social world. According to Jenkins, identity is not only about individual values and understandings, but also about interactions through which status, social position, roles and expectations become visible. Furthermore, Jenkins refers to identity as a process rather than a static constant.

${ }^{\mathrm{B}}$ In line with the work of other authors, ${ }^{8-10}$ risk in the context of our study is experienced and handled in an area of tension between objective and subjective. ${ }^{9}$ From a medical/statistical perspective, risk is the probability for a certain person to develop FBOC, based on the outcomes of genetic testing. From a socio-anthropological point of view, risk can be considered as a relational phenomenon that is situated in specific social and cultural contexts. As a way to unravel these "juxtapositions and amalgamations" of perspectives, Boholm proposes considering risk "as a cognitive frame that produces contexts which link an object of risk (a source of potential harm), an object at risk (a potential target of harm) and an evaluation (implicit or explicit) of human consequences;" situated in specific social contexts risk can be thought of as a relational order through which connections between people, 'things' and 'outcomes' are constituted." This understanding of risk also encompasses the varying perspectives of different actors on the same external phenomena; ${ }^{10}$ and it is within this cognitive frame that persons confronted with the statistical probability of getting $\mathrm{FBOC}$ are required to make their evaluations and to negotiate their individual risk perceptions. social and political engagement concerning health-related issues). ${ }^{13-15}$ The number of publications under the heading of HL has risen rapidly in recent years, and efforts continue to define the concept, to expand existing definitions, or to examine them in new contexts. ${ }^{16}$ In the phase of conceptualising this study, we employed a working definition of HL based on the existing literature and the integrated model of HL, defining it as a person's set of certain abilities, attitudes and lifestyle, enabling her or him to manage a certain health situation in order to achieve a positive health outcome. ${ }^{10}$

A great number of studies deal with the measurement and quantification of $\mathrm{HL}$ as a multidimensional concept. The majority of them primarily refer to functional HL using standardised measurement tools and mixed measurement (self-reporting components and direct testing of certain abilities). ${ }^{17,18} \mathrm{~A}$ few very fruitful qualitative studies deserve closer attention ${ }^{19-21}$ that analyse HL beyond its functional dimension, using data from interviews and thereby emphasizing the perspective of those being studied. However, qualitative research on HL to date is still scarce, particularly in the specific clinical field of risk of FBOC. ${ }^{22,23}$ At this point in time, we still know very little about people's own perceptions of HL in the context of FBOC and of factors relevant for the management of health-related information and behaviour. An insight into the process of decision-making, attitude towards risk, and the individual coping strategies before, during and after the genetic testing can provide a productive ground for a better understanding of $\mathrm{HL}$ in the context of risk.

The current study aims at generating new hypotheses in the research field of HL and risk based on findings from a bottom-up research approach. The leading questions in this article are: i) Which factors account for HL among persons at risk of developing FBOC? ii) How can the empirical findings contribute to the theoretical foundation and conceptualisation of HL?

\section{Methods and research ethics}

This study is part of a broader research project on HL (Health Literacy of Persons at Risk - From Information to Action - RisKomp) in persons at risk in four different clinical fields (FBOC, coronary heart disease, psychosis, and Alzheimer's dementia) and uses a qualitative design integrating an ethnographic-narrative approach. This article is based on findings from 10 narrative,,$^{24}$ open-structured interviews with women who have undergone genetic testing for FBOC. Ethics approval was obtained in March 2018, (registration number 18-014) by the ethics committee of the Medical Faculty of the University of Cologne.

\section{Procedure and participants}

The interviewees were recruited at the Centre for FBOC at the University Hospital of Cologne (Germany) 
in cooperation with a team of experts in the field of FBOC and genetic testing according to the criteria shown in Table 1 . The team of physicians closely involved in the clinical everyday practice and procedures started screening patients' data for eligibility and obtained the patients' agreement to be contacted by the research team. Only individuals who underwent genetic testing were contacted; those who did not, were not included in this study. The researchers arranged the meetings and conducted the interviews. All participants were provided with detailed information on the aims of the study and the research procedure and signed an informed consent for participation. In a time period of five months, all 10 interviews were conducted and preliminarily analysed; in the next six months, all interviews were transcribed and went through an in-depth analysis process.

\section{Narrative interview}

The interviews started with an opening question avoiding a direct reference to feeling about risk and rather focusing on the concrete situation in which the first confrontation with the notion of genetic risk happened. Further in-depth questions were pre-formulated based on existing empirical research and were used only if the topic was raised by the interviewee. An additional tool was developed to ensure a constant process of researchers' selfreflection, researcher triangulation, and making researcher's subjectivity visible. The average length of the interviews was approximately one hour. All interviews were conducted in German. For the purpose of this article, the quotations from the interviews used in the analysis part were translated from German into English by the involved researchers including the one who conducted the interview. The different versions of the translation were compared, discussed, and in case of disagreement, a fourth person (also a member of the team but not involved in the research process) was asked for her/his opinion. Socio-demographic data were collected by means of a questionnaire before starting the interview.

\section{Analysis strategy}

The process of data analysis was embedded in the reflexive grounded theory, ${ }^{25}$ a variation of the grounded theory methodology emphasizing the interaction between the researcher and the research topic/partners/or field as an in- tegral part of the process of knowledge production and considering reflection on this interaction as a source of insight. It combines the grounded theory approach with reflexivity concerning the situatedness of the researchers, the research design, as well as the research process and practices. Reflexive Grounded Theory therefore allows for the integration of the researchers' subjectivity, personal dispositions, or standpoints into the analysis, as well as for the reflection on power relations in the research context.

The data analysis in the current article is based on two data pools - the interviews and the researchers' self-reflection - with the aim of ensuring a comprehensive analytical process encompassing reflexivity, theoretical sensitivity, and triangulation of data and researchers. All researchers and participants in this study were female. The researchers' own understanding of risk grounds in their different academic backgrounds (psychology, health sciences and social anthropology) and in their personal experiences with risk and disease. The three researchers had also been confronted with the risk of getting FBOC to different extents in different phases of their lives and made different decisions on prediction and prevention. These experiences and attitudes have undoubtedly impacted the research process; in line with the principles of the reflexive grounded theory, they were actively addressed throughout all stages of data collection and analysis.

In the first stage of the empirical research, data were analysed parallel to the process of conducting the interviews. Our team arranged researcher meetings on a regular basis and created opportunities to discuss and compare the first impressions and categories that resulted from the first steps of individual open coding. Inductive thematic saturation was reached at the sixth interview. ${ }^{26}$ In the second stage of the analytical process, the memos were systematically analysed, and the findings were incorporated into the process of axial coding. In the third stage of the analysis, the two data sources were integrated into a process of selective coding and theory generation.

\section{Participant characteristics}

The analysis includes responses of ten women: two women aged between 18 and 30 (Julia, incapacitated for work and Kathrin, working part-time in the health area); four women aged between 31 and 40 (Christina, working part-time in the social area, Jennifer, working fulltime in

Table 1. Inclusion and exclusion criteria.

\begin{tabular}{ll}
\hline Inclusion & Exclusion \\
\hline Participation in genetic counselling taken place & Persons younger than 18 years of age \\
Group 1: Carrier of a BRCA1 or BRCA2 mutation & Mild cognitive impairment or dementia \\
Group 2: Carrier of a mutation in a moderate risk gene (e.g. CHEK2) & Current clinically relevant depressive episodes, anxiety \\
Group 3: No mutation detected in one of the known risk genes, but increased & symptoms or suicidal tendencies \\
statistical risk of disease due to own and/or family anamnesis & \\
Written declaration of consent of the patient & \\
German language proficiency that allows for participation in an interview/survey & \\
\hline
\end{tabular}


the health area, Stefanie, working part-time in the field of economics, and Nadine, working in the health area); three women aged between 41 and 50 (Katharina, working full-time in the social area, Sarah, working fulltime in the IT area and Anna, working full-time), and one woman aged between 51 and 60 (Sabrina, working parttime in the health area). Six of the participants were married, three unmarried, and one divorced. Most participants lived with their families or partners, apart from one who lived alone. Seven participants had a German background and three, a non-German cultural background. All participants had secondary to post-secondary educational background. Four participants suffered from a chronic disease, e.g. thyroid gland diseases. All names used in text are pseudonyms.

\section{Category system and main findings}

The main themes and issues that arose in the course of this study can be situated in the context of understanding risk, identity (being a person at risk of developing FBOC), and managing health. These spheres also build the central categories of analysis, contributing to a better understanding of $\mathrm{HL}$ in persons at risk of developing FBOC.

\section{Understanding the genetic risk of getting ill - as a feeling, a multifaceted concept, and an individual/relational responsibility}

The process of understanding health risk in its different dimensions includes the individual's feeling about risk, its familial dimension, and possible strategies to manage and control the process of risk understanding. This negotiation also entails questions about the credibility of risk when related to everyday life experiences.

\section{Risk as a feeling: between reason and emotions}

The results of this study indicate a tension between different interpretational frameworks concerning one's genetic risk: a logical framework, analysing risk based on statistics and medical facts; and an intuitive one, viewing risk on a more affective level through one's own experiences. ${ }^{8}$

With respect to the appraisal of a genetic risk of FBOC, this complex interplay between both frameworks is mirrored in our interviewees' accounts. ${ }^{8}$ On the one hand, the demand for genetic testing as a means to objectify risk and as a basis for further decision-making on prevention suggests that in medical settings, reason (representing the analytical system) is the dominant mindset among both professionals and patients. On the other hand, our interviewees' narratives demonstrate the importance of emotions (representing the experiential system) in the process of interpreting and evaluating risk on an individual level. The decision for genetic testing was described with an emphasis on the role of one's own risk-intuition, and at the same time as a possibility to tech- nicise risk and thereby attain a sense of safety. Sabrina responded to the question of how she was confronted with the risk of getting FBOC for first time saying:

I somehow knew that I have a high risk, and then I complained to my gynaecologist, and I said to her: I think that I'm quite at high risk, I want to do the test, and then I also wish to act accordingly. And then they said: You do not need to do the test, your cousin is not affected, the youngest and most severely affected family member. We have examined her tissue, and there is no gene. (PALP2, breast cancer)

The interviewed women reflect on their endeavour of relating the medical information about the statistical risk probability to their personal feelings towards the risk (in terms of a premonition or an intuitive interpretation of the statistics they have been provided with). When being asked how she values the communicated risk percentages, Jennifer explains this as follows:

I myself for example, from a purely medical point of view, I understand that they say that you only consider it a medium risk if it is increased up to 40 percent. But for me personally, it felt like close to 50 percent and the chance is actually fifty-fifty whether you will get it or not. (CHEK2, breast cancer)

This quotation illustrates the process of relating medical information to one's own feelings. Our interviewees' reflections suggest that the two sources of information (numbers and feelings) are not merely considered a problem of contradiction, but also as a resource for decision-making. This shows that understanding the quantity of risk correctly in mathematical terms and feeling about its quality may be seen as complementary rather than contradictory.

\section{Risk as a multifaceted concept}

The depiction of risk in an area between the emotional and the rational is also related to one further perspective of understanding risk and decision-making, namely experiencing risk in manifold facets. These were described with respect to i) illness-logic, i.e. the observation that whether or not someone will be struck by a disease is not always logical in terms of risk factors; ii) inevitability, i.e. the awareness that one cannot influence the fact of having or not having inherited the gene; and iii) a call to action, i.e. the responsibility that one may be able to influence whether or not the gene will eventually result in developing the disease. All our interviewees provided many examples for the paradox of risk in terms of the illness-logic in order to substantiate their decision to listen to their individual feeling. For example, Julia refers to this paradox while responding to the question of how she became aware of the risk saying: 
Well, I knew it before. That sounds stupid, but my grandfather died of cancer, but he was the healthiest man ever-he never smoked and stuff, or drank. (BRCA2, breast cancer)

The interviewed women also discuss the pathogenesis of FBOC emphasizing its paradoxical nature between determinism and modifiability: Sabrina sees it as a genetic bug and one's own genes as an uncontrollable danger, and Julia as variable, modifiable by stress and way of life:

Well, you feel as if you were carrying a time bomb with you. (Sabrina, $P A L P 2$, breast cancer)

But I just think, somehow, considering how fastmoving society is, and the pressure you feel in working life and so on, people just get sick.... (Julia, BRCA2, breast cancer)

\section{Individual and relational responsibility}

The feeling of risk in the context of FBOC is a topic which also implies a further relational/familial dimension. The possibility for genetic testing is seen as emanation of the individual's autonomy, on the one hand, and on the other hand, it raises the topic of the distressing effect on the tested person and her/his family and relatives if the result is positive. Asked about her experience with genetic testing, Sabrina said:

And there is a constant back and forth, right? That you say: I do not want to know it, it is driving me crazy. You almost feel like it's a self-fulfilling prophecy. (PALP2, breast cancer)

In this quotation, the notion of responsibility and self-fulfilling prophecy also becomes visible. Women reason on the relation between being overconcerned with risk and the illness outbreak, wondering if they themselves could somehow influence its emergence. Responsibility is not only a question concerned with the individual and her impact on the disease emergence, but also with her as a part of the familial system. Christina provides an example for this familial dimension when asked about how her family deals with the results from genetic testing:

My mother even apologised to me for transmitting the gene to me. But that is not at all her fault.

(BRCA2, no cancer)

In this case, the genetic risk goes beyond the individual responsibility (in terms of behaving in a way to minimise it) and reveals a further collective dimension. Christina's genetic risk is immediately intertwined with her mother's risk and raises the question of fault, responsibility, and the distribution of blame.

\section{Strategies to manage risk}

The different biographic backgrounds influence the strategies the interviewees employ in order to manage the thought about possible sickness in the future or acute onset of a disease in the present. Factors that influence the perception of illness are: i) vicarious experience as in the case of Sabrina, ii) the perception of illness as a normal and universal part of human life as described by Julia, and iii) medically related work experience in the case of Jennifer:

So, and this horror scenario was something I had then, in the same way, like the images of my mother who died so terribly and then I thought: I have to do it in an extreme fashion [the prevention]. (Sabrina, $P A L P 2$, breast cancer)

And if you look into families, there is hardly any family, I would say, without any diseases, or strokes of fate $[. .$.$] I see diseases as everyday risk."$ (Julia, BRCA 2, breast cancer)

So... and we've had such a chief physician whom I have to think about all the time. I am actually working in surgery. And he always used to say, he always was in favour of resecting generously. (Jennifer, CHEK2, breast cancer)

To sum up, the concept of risk as a feeling in the area of tension between emotions and reason is seen as an important aspect in the processes of perceiving risk and decision-making. Describing risk as a multifaceted concept giving examples based on personal, everyday experience performs an argumentative function in explaining one's own attitude and serves as evidence that medicine/statistics/science are not universal. The question of responsibility is seen in both an individual (doing something that could trigger the disease) and a social (genetic responsibility) dimension.

\section{Identity between quality of life, social environment, everyday life, and stress}

This category illustrates the role of personality in the process of dealing with risk and, at the same time, the impact of risk on one's identity ${ }^{3}$ and life-world. ${ }^{27}$ Women describe their quality of life in a close relation to their personality, social environment, everyday life, and their individual ability to regulate and control stress.

\section{Quality of life}

In terms of quality of life, it became clear that the personality determines the individual, subjective definition of the concept. Katharina responds to the question of how she manages her risk saying:

Well, I'm not in the mood for side-effects [of the preventive medication], if that's what's affecting 
the quality of life. I want to live as well as I can. I want to do sports, make music, work [...]. (ATM, breast cancer)

When asked about their understanding of health, Anna and Jennifer responded referring to the term "quality of life." On the one hand quality of life is seen as condition that should be actively maintained in doing something spontaneously and actively enjoying life as in the case of Jennifer; on the other hand, it is defined by keeping up with normal life and feeling able to live in the normal way as explained by Anna:

I need to take better care of myself and that I also just do such spontaneous things just more often, you know? That I just say: Nope. I have the weekend off and my husband is on duty, so I'm just going to Holland to the seaside, just like that, you know? (Jennifer, CHEK2, breast cancer)

That is, being healthy for me means being able to lead a normal life. That includes work, it includes friendship, it includes family, it includes my partner, it includes sports. Being able to join cultural things, you know? So that I can do all of this. I can lead a completely normal life. And for me in a way that just means being healthy. (Anna, $C H E K 2$, breast cancer)

In Anna's words, normality relates to the life before knowing about the risk or having cancer, and quality of life means to maintain this condition, not allowing risk and illness to take up too much space and thereby endangering the normal life. In the case of Jennifer, the risk and the illness can be seen as a ground for actively increasing the subjective quality of life through enriching the normal life by spontaneous actions and experiences.

\section{Social environment and everyday life}

The concerns related to the concept of quality of life are connected with the feeling of anticipated loss of everyday-life normality, especially in the context of life planning and decision-making with respect to one's own self or in the impact on relatives and family members, as in the case of Jennifer. Asked how knowing about the risk change her life, she responded saying:

So, I thought: It will never be the way it was, and you will never get back your normal life. And I found that really bad. And so just to have back this normality. And yes, the normality is another one now, but also good. (CHEK2, breast cancer)

The knowledge about the results of the genetic testing is also seen in close correlation to everyday-life normality and social relationships. For example, Katharina reports feeling the predicament of keeping something (the information about risk) for herself, as a secret towards children, parents, or other family members, and to the impact which this has on their social environment:

Well, you can't always talk about everything [when the children are there], can you? (ATM, breast cancer)

\section{Dealing with stress}

Another important factor connected to the preservation of quality of life and normality is the concept of stress. The strategies to balance stress in everyday life build the core of understanding quality of life and the concept of HL, which will be described further below. One strategy of stress regulation is to adapt one's personal attitude towards life circumstances by, for instance, becoming more relaxed. Stefanie responded to the question of how does the knowing about risk impact her everyday life saying:

Just to slow down a little, to be a little bit more relaxed and, you sometimes get to work on that, but it is difficult to put it into practice. (BRCA2, no cancer)

A further strategy to balance stress is related to the interaction with others and training one's own ability to put personal capacities into centre as in the case of Sabrina:

I am allowing myself more time for things; these stressful things where I used to think "I have to, I have to, I have to" - these are more indifferent to me now." (PALP2, breast cancer)

Additionally, Christina, for example, has also identified and employed stress management strategies in everyday life that are connected to certain activities, habits, and behaviour:

Yeah, somehow not to have so much stress all the time. Exercising every day and paying attention to healthy eating, also for my kids. Then... well. Then you also realise that life is short and that you ought to make the most of it as best as you can. (BRCA2, no cancer)

The findings of this study show that the concept of quality of life is primarily seen in its individual and subjective dimension, being used as a synonym for normal life. Regulating stress inwards through one's own attitude and outwards in the interactions with the social environment is seen as a key factor for preserving quality of life in the context of genetic testing.

\section{Managing health on the crossroad of information, self-reflection, and expectations towards the system}

Information and self-reflection, expectations towards the system, and role expectations and definitions in the 
doctor-patient relationship emerged as central subcategories with respect to the process of managing health.

\section{Information and self-reflection}

When asked about how they inform themselves with respect to risk and FBOC, the interviewed women discussed and reflected on their personal strategies of searching for and finding relevant and trustworthy sources of information. For example, Sarah outlined her motivation of getting information in addition to the regular doctorpatient conversations and Kathrin advocated her need or decision to look up information on the Internet:

[Regarding the search for information on the Internet] Maybe that wasn't quite right, either. But, well, as I said, I thought the more I know, the more I'm in control, and the better that works. But, as I said, it's just, I think that is just such a way one could deal with it, as it were.... (Kathrin, familial cancer history, no cancer)

Yeah, that's the way it is, you need to critically question everything. I know there are quite a few scientific publications that are not based on real studies. [...] However, when I see the websites run by German clinics, by the university hospitals, I can rely on those, since, well, if even a university hospital spread rubbish, that would just be.... (Sarah, familial cancer history, no cancer)

These quotations demonstrate the women's awareness that the Internet as an information channel needs to be consulted with caution and their effort to justify the way they make use of it. Apart from that, Kathrin and Sarah describe a feeling of being unsure and timid in this information process, accentuating the complicated way information is being written as well as difficulties in finding universally correct medical information.

\section{Expectations towards the system}

This subcategory evolved in a close relation to the topic of decision-making for risk prediction. In this context, the interviewed women discuss and evaluate the doctor's recommendation of risk-controlling measures, on the one hand, and emphasize the importance of the feeling of making autonomous decisions, on the other. In retrospect, Kathrin, for example, reflected on whether the outcome of the genetic testing had met her expectations and helped her decision-making:

Well, and in the end, we are human beings and not statistics [...]. I first thought, Well, okay. Maybe we will manage that in one way or another. And then, nope, [the testing] has not brought me any certainty on no account. It brought me, so I think, uncertainty, and in retrospect, at some point, I thought, Let's wait and see, what will be, will be, and before that you cannot do anything anyway. (Kathrin, familial cancer history, no cancer)

Nadine responded to the question concerning her experiences with doctor-patient communication describing her ideal of the doctor's role as empathic, competent, and able to communicate at eye-level:

I have the feeling that my actual gynecologist is not an incredibly well-informed doctor in this area, because he has a different specialty. But what helped me most was the counselling center for FBOC in the university hospital. I have the feeling that my questions have been answered. And if I have a question, I will go there again. (BRCA1, breast cancer)

Asked the same question, Katharina responded thematising her awareness of the role of self-proclaimed "annoying patient" and describes her strategies of how to avoid being seen as such by her doctors:

You [as a doctor] have to talk to people. I don't want to be annoying either, but I just want to be taken seriously and get answers, right? (ATM, breast cancer)

Women also thematise the power relation in the doctor-patient communication and reveal their strategies to cope with it i) by actively reflecting the power constellation and asking questions (in the case of Katharina) and/or ii) by asking for a recommendation on how to decide, adopting the passive role in the communication process (as in the case of Jennifer):

And nowadays, it is often still like that, the doctor is way up high and the patient has to, as it were, like obey or so. (Katharina, ATM, breast cancer) But you really are, you want to, well, the perspective is really a different one and you just want that other people, so, that the doctors decide on your behalf. Well, I could not have made many of the decisions by myself. (Jennifer, CHEK2, breast cancer)

The ability to manage health and risk is seen as the competence to find relevant information and adapt it to the individual context in a constant process of self-reflection, critically estimating whether a certain recommendation fits into one's own life-world or not. The concept also includes a systemic dimension manifested in the doctorpatient relationship - being aware of the role-attributions that professionals assign to patients, being able to formulate one's own role-expectations towards professionals, and working out strategies to manage and reflect on this power-constellation. 


\section{Discussion and implications for practice}

The findings of this study allow for insight into different levels of knowing about, perceiving, and dealing with an increased risk of developing FBOC. The women's narratives show the entanglement of managing one's health, the risk of getting ill, and one's identity, as manifested through the individuals' description of their risk perceptions. In the following, we will first discuss these risk narratives, interpret their meaning in terms of $\mathrm{HL}$, and then relate our empirical data to existing concepts and theories of HL, discussing possible implications for research and practice.

\section{The meaning of health risk in the context of FBOC}

Our study revealed the intertwining of meaning-making with respect to one's risk and HL. In our interviewees' narratives, a health risk turns into a risk of getting ill or something that can let the illness break out and develop. This shift in the meaning of risk speaks for an increased awareness of the possibility of getting breast or ovarian cancer and a decreased risk of the possibility to maintain the condition of not having cancer in the present and future. In the case of individuals with no manifest cancer disease, this can also mean a shift in the self-attributed role definition -from a healthy individual to a patient or carrier of hidden genetic thread. As Gunn et al. have demonstrated in their study on women at risk of developing FBOC, it is essential to sharpen health communicators' sensitivity for the risk explanation models of advice seekers. ${ }^{28}$ In the following discussion, we will address three conclusions regarding interviewees' risk perceptions and their impact on HL in the sense of a person's management of health information and health behaviour.

\section{Feeling about and understanding of risk as a multifaceted concept}

Our findings show that the appraisal of risk has a dualistic nature: intuition is seen as a trustworthy source of risk awareness; at the same time, it gets meaningful only if it attains the status of factual knowledge, providing some extent of scientific value that can be quantified, measured, and observed. The interviewed women see risk as a theory of probabilities and statistical data that should be taken seriously, but at the same time they oppose these objectivity claims to their everyday experiences. These findings can be discussed within the framework of Slovic et al.'s theory of risk, giving us more information about the way individuals realise, perceive, and negotiate risk within their experiential system, questioning the attributed value of its accurate numerical perception. ${ }^{8}$ This underlines that risk literacy is much more complex than simply understanding risk in correct statistical terms. Our findings draw a specific picture of risk in the context of prevention, locating genetic risk in closer proximity to disease than to health; genetic predisposition is perceived as a dormant disease that can break out under certain conditions. With a view to the counselling practice, we recommend reserving a definitional space for discussing genetic risk vs. disease (incorporating patients' explanatory models) in the patient-doctor communication and genetic counselling.

\section{Genetic testing as a signpost and as a trigger for individual risk management}

According to our findings, intuition is often the reason for choosing genetic testing, and the testing procedure is seen as a way to technicise one's intuition and give the subjective feeling of fear and uncertainty an objective, medically approved face. Dealing with risk consists of renegotiating its dualistic nature in each individual situation of decision-making and in the construction of a person's individual life narrative before and after genetic testing. With respect to healthcare practice, these findings can offer a fruitful ground for future work in the sphere of genetic counselling and communication, delivering focal points for more patient-centred communication by addressing the individual's lived experiences, identity, and life-world.

\section{Risk in a close relation to (hypothetic) disease perceptions}

The ideas about the potential disease may be crucial for dealing with and interpreting risk. ${ }^{22}$ These often arise based on past experiences of others (often family members, friends, or patients if the person works in healthcare). In addition, the understanding of disease as a phenomenon varies between a universal dimension, being seen as an almost normal condition (all people have some disease), and a personal dimension, considered as a particular threat due to one's own risk genes. This DNA fraught with risk is, on one hand, seen as a trigger or even an early stage of the disease, and, on the other hand, as a matter of a person's own responsibility. As a recommendation for counselling practice, both the perception of the potential illness and the topic of individual responsibility (not only in terms of agency, but also as a possible burden) should, in our opinion, gain more relevance in the patient-doctor-communication.

\section{Health literacy in the context of risk - different faces and diverse facets}

The above outlined facets of risk perceptions among individuals who have undergone genetic testing in order to learn more about their risk of getting FBOC open up a new possible perspective of approaching the concept of HL. In the following, we will embed our findings into three conceptual dimensions of HL discussed in the recent literature. 


\section{Health literacy between personal and relational concerns}

The findings of our study mirror the academic discussion on HL, defining it as an individual set of abilities, attitudes and lifestyle, ${ }^{16}$ evolving within a specific health/disease situation, ${ }^{20}$ manifested through and formed by communicative practice. ${ }^{29} \mathrm{We}$ argue that in the context of risk, HL should be understood in both its individual and relational dimension, emphasizing its meaning as communicative action. ${ }^{30}$

As the results of our study show, the way individuals deal with health risks is strongly influenced by their own identity. The personal understanding of quality of life, the role of stress, and the social environment have an impact on how individuals deal with risk and illness. Similar to the findings of Sanders et al. ${ }^{31}$ our study shows that being an observer of someone else's suffering leads to the projection of these impressions and fears on one's own possibility to become ill and thus forms the understanding of health-oriented behaviour. This entanglement between the individual and the collective/relational dimension of the concept of HL leads us to ethical issues regarding the fine line between agency and genetic responsibility. Our results are in line with existing studies, ${ }^{12,32}$ showing that genetic risk is seen not only in its medical relevance in terms of prediction and prevention among family members, but also as a factor influencing social and family relationships. Referring to this, we strongly support the call by Maddock et al. ${ }^{33}$ for improvements in risk communication, emphasizing the need of paying attention to the topic of telling the family. In the counselling practice this may imply the use of strategies to increase awareness for the relational responsibility in a familial context, providing recommendations for communicating risk to family members, and encouraging individuals to engage in reflections on the consequences of knowing about risk for themselves and for their family members. On an organisational level, it may be helpful to integrate and further develop complementary family counselling.

\section{Health literacy as interdependence between individual and (health) system}

$\mathrm{HL}$ is mostly seen as an individual-centred concept which means that individuals apply a set of abilities and attitudes to manage health information in a concrete health/disease situation in order to achieve the best possible health outcome. ${ }^{16}$ Nevertheless, a striving for enhancing the organisational/systems-related dimension of the concept can be observed in the academic field. ${ }^{34}$ In our study, this dimension becomes visible through the description of the expected roles of doctors in the consultation and healing process.

Holmberg et al. found that the most important factor for deciding on the intake of a certain medicine for the treatment of breast cancer was the doctor's recommendation. ${ }^{35}$ On one hand, this can mirror trust in the search for orientation in an ambiguous situation. At the same time, it may also demonstrate the power gap in an existentially threatening decision-making situation. For example, Dixon-Woods et al. have shown, in their research among women who consented to surgery in obstetrics and gynaecology, the weakness of the consent process as a safeguard of autonomy (p. 2742) in the context of hospital structure and rules of conduct. ${ }^{36}$ Our findings suggest similar mechanisms in the case of decision-making on risk prediction and preventive interventionsboth the impact of doctors' recommendation on a certain preventive decision and the felt power gap in the consulting situation, emerged as relevant categories during the research. Nevertheless, our study does not provide enough data to draw general conclusions about shared decision-making in the context of risk, and, hence, calls for further research on this topic.

According to our findings, the concept of stress appears to play a strategic role in the process of coping with health risk. Our analysis showed that our interviewees address stress both as an alert system enabling a successful risk control and, at the same time, as an external and internal source of hidden and uncontrollable danger. While the internal danger is associated with the feeling of anxiety, the external one is closely related to stress caused by the health system and can be discussed in the wider context of organisational HL. While the health system is seen as a source of healing in terms of risk control, it is also perceived as a source of stress that can provoke a disease breakout. Being health literate, from the perspective of the person at risk, in this context not only means complying with the health system and correctly understanding risk information, but particularly finding an individual balance to regulate both sources of stress. From the perspective of organisational $\mathrm{HL}$, it is worth addressing the question of how a system can reduce stress in the case of genetic prediction and how it can provide a supportive environment for individual HL.

Our study provides some focal points for understanding $\mathrm{HL}$ in its communicative dimension ${ }^{29}$ this is in line with the findings of Sarangi et al. ${ }^{37}$ who describe risk assessment and management as a dynamic process of negotiation between advice seekers and professionals, thereby co-constructing and putting into perspective the meaning of risk. We strongly believe that for research and counselling practice it will be fruitful to pursuit further research on possible tools and instruments that may support the process of interaction as a space where individual and system meet, and that could contribute to the evolution of a joint, collaborative HL.

\section{Critical health literacy as a resource for risk savvy}

The interpretation of the risk narratives in the context of FBOC and their relation to the concept of HL discloses one further crucial dimension of handling health information on risk - critical HL as the ability to assess 
the credibility and applicability of the risk information and to challenge its meaning. ${ }^{13}$ The findings of our study suggest that it is closely intertwined with quality of life; in particular, with regard to the critical appraisal of information and the ability to assess its personal relevance. ${ }^{13}$ In the context of our research, critical HL becomes relevant in the process of relating health information to one's subjective quality of life. The women's narratives reflect an attempt to carefully select and apply those pieces of information which do not interfere with their quality of life in an inacceptable way and, hence, outweigh the potential health benefit. While the sacrifice for the future and the loss of the feeling of not knowing is seen as a justified sacrifice in the name of life, the sacrifice in the present is seen as burden and a cut in the individual quality of life. The delicate trade-off between benefits and harms, expected gain and taking a loss was also discussed by Bloom et al. with respect to prophylactic mastectomy; they found that women were ready to pay an unexpectedly high price (i.e., sacrificing their breast) for a relatively small reduction of risk. ${ }^{38}$ Based on their findings, the authors recommend that instead of putting risk statistics at the centre of communication, physicians should rather emphasize patients' lived experiences in order to support them in being prepared for the emotional and physical consequences of their decision. With respect to the practice and in line with this recommendation, we argue that the promotion of critical HL may enable an individual not only to critically analyse information, ${ }^{14}$ but also to incorporate the identity (attributed roles and expectations), life narratives about health and risk, familial relationships, and everyday situations which may be influenced by the knowledge of genetic risk or the distress before testing. This is why we believe that it might be a winning approach to search for ways of promoting critical HL in the counselling practice, embracing the concept of subjective quality of life, and integrating it into institutionalised communicative action.

To sum up: The genetic testing is not an event that triggers and forms patients' attitudes towards the questions of risk, health and illness. It is much more a process that brings these attitudes, values, and ideas to the surface. This is the reason to suggest that patients' explanatory models of risk, their general attitude towards health and disease, their lived experience ${ }^{39}$ and biographical background should be taken into consideration in the course of genetic counselling in order to promote both individuals' and doctors' HL.

\section{Methodological reflection, strengths, and limitations}

Limitations on two different levels - setting and methodology - need to be discussed in the context of this study. The definition of inclusion and exclusion criteria was chosen in close agreement with medical experts according to the current state of medical evidence concerning risk prediction in the field of FBOC. In this way, persons who believe to be at risk without having a medical proof of their intuition in terms of genetic testing were excluded from our study. Hence, the composition of our sample does not unrestrictedly mirror the claim "to focus on the subjective perceptions of risk and HL." Apart from that, we also excluded persons who had been recommended to participate in a genetic testing and decided against it. In addition, in the case of FBOC, like for many other conditions, a clear demarcation between not at risk, at risk, and ill is not always possible (e.g., in the case of women who had breast cancer and underwent a genetic testing for the healthy breast). Hence, a differentiated definition of diverse at-risk conditions to be eligible for our study was needed. Based on our experience, we therefore recommend considering time for discussion and agreements with the respective clinical partners.

\section{Conclusions}

Given the increasing discussions and critical voices concerning the theoretical anchoring and conceptualisation of $\mathrm{HL}$ and the large variety of its existing definitions in recent years, the findings of this study suggest some complementary cornerstones for theory generation based on qualitative data, approaching HL from a bottom-up perspective. In our opinion there is a need for more explorative, collaborative, and interdisciplinary research in this field. We, therefore, call for an alternative and more holistic approach to HL in science, medical practice and research, and the development of tools that address both provider and recipient of health services at once.

Risk prediction in the context of health and disease is always a call for action and can have positive, but also negative, consequences for an individual's life. In the case of genetic risk, it is not only a call for action for the affected person, but also for relatives and family members. In this context, the relational, collective dimension of HL is crucial for the better understanding of the concept and needs to be considered when developing HL promoting interventions.

Technical innovations in the field of genetics and predictive medicine will beyond doubt lead to the detection of an increasing number of risk factors. This will present HL research, politics, and public health for the next generations with the challenge of mediating this ever-expanding medical field with corresponding considerations concerning decision-making, prevention, and stress management in the view of persisting statistical uncertainty. We, therefore, believe that a definitional space needs to be reserved for discussing genetic risk vs. disease, incorporating patients' explanatory models in patient-doctor communication and in genetic counselling. 


\section{References}

1. Rhiem K, Bücker-Nott H-J, Hellmich M, et al. Benchmarking of a checklist for the identification of familial risk for breast and ovarian cancers in a prospective cohort. Breast $\mathrm{J}$ 2019;25:455-60.

2. Hauke J, Horvath J, Groß E, et al. Gene panel testing of 5589 BRCA1/2-negative index patients with breast cancer in a routine diagnostic setting: results of the German Consortium for Hereditary Breast and Ovarian Cancer. Cancer Med 2018;7:1349-58.

3. Jenkins R. Social identity. 2nd ed. London: Routledge, 2014.

4. Appleton S, Fry A, Rees G, Rush R, Cull A. Psychosocial effects of living with an increased risk of breast cancer: an exploratory study using telephone focus groups. Psycho-Oncology 2000;9:511-21.

5. Caiata-Zufferey M. Genetically at-risk status and individual agency. A qualitative study on asymptomatic women living with genetic risk of breast/ovarian cancer. Social science \& medicine 2015;132:141-8.

6. Bluman LG, Rimer BK, Regan Sterba K, et al. Attitudes, knowledge, risk perceptions and decision-making among women with breast and/or ovarian cancer considering testing for BRCA1 and BRCA2 and their spouses. Psycho-Oncology 2003;12:410-27.

7. Kenen R, Arden-Jones A, Eeles R. Healthy women from suspected hereditary breast and ovarian cancer families: the significant others in their lives. Eur J Cancer Care (Engl) 2004;13:169-79.

8. Slovic P, Finucane ML, Peters E, MacGregor DG. Risk as analysis and risk as feelings: some thoughts about affect, reason, risk, and rationality. Risk Anal 2004;24: $311-22$.

9. Boholm $\AA$. The cultural nature of risk: can there be an anthropology of uncertainty? Ethnos 2003;68:159-78.

10. Boholm $\AA$, Corvellec H. A relational theory of risk. J Risk Res 2011;14:175-90.

11. Klitzman R, Chung W. The process of deciding about prophylactic surgery for breast and ovarian cancer: patient questions, uncertainties, and communication. Am J Med Genet 2010;152A:52-66.

12. Etchegary H, Miller F, deLaat S, Wilson B, Carroll J, Cappelli M. Decision-making about inherited cancer risk: exploring dimensions of genetic responsibility. J Genet Couns 2009; 18:252-64.

13. Chinn D. Critical health literacy: a review and critical analysis. Soc Sci Med 2011;73:60-7.

14. Nutbeam D. Health literacy as a public health goal: a challenge for contemporary health education and communication strategies into the 21 st century. Health Promot Int 2000;15:259-67.

15. Liu C, Wang D, Liu C, et al. What is the meaning of health literacy? A systematic review and qualitative synthesis. Fam Med Community Health 2020;8:e000351.

16. Sørensen K, van den Broucke S, Fullam J, et al. Health literacy and public health: a systematic review and integration of definitions and models. BMC Public Health 2012;12:80.

17. Altin SV, Finke I, Kautz-Freimuth S, Stock S. The evolution of health literacy assessment tools: a systematic review. BMC Public Health 2014;14:1207.

18. Haun JN, Valerio MA, McCormack LA, Sørensen K,
Paasche-Orlow MK. Health literacy measurement: an inventory and descriptive summary of 51 instruments. J Health Commun 2014;19:302-33.

19. McKenna VB, Sixsmith J, Barry MM. A qualitative study of the development of health literacy capacities of participants attending a community-based cardiovascular health programme. IJERPH 2018;15.

20. Samerski S. Health literacy as a social practice: Social and empirical dimensions of knowledge on health and healthcare. Social Sci Med 2019;226:1-8.

21. Jordan JE, Buchbinder R, Osborne RH. Conceptualising health literacy from the patient perspective. Patient Educ Couns 2010;79:36-42.

22. Rutherford EJ, Kelly J, Lehane EA, et al. Health literacy and the perception of risk in a breast cancer family history clinic. Surgeon 2018;16:82-8.

23. Ektir B, Yilmaz M. Breast cancer-related knowledge levels and health literacy in working women. Int J Caring Sci 2017;10:1658-68.

24. Nohl A-M. Interview und Dokumentarische Methode. Wiesbaden: Springer, 2017.

25. Breuer F, Muckel P, Dieris B, eds. Reflexive grounded theory, Eine Einführung für die Forschungspraxis. 4th ed. Wiesbaden: Springer, 2019.

26. Saunders B, Sim J, Kingstone T, et al. Saturation in qualitative research: exploring its conceptualization and operationalization. Qual Quant 2018;52:1893-907.

27. Schutz A, Luckmann T. Structures of the life world. Lodon: Heinemann, 1974.

28. Gunn CM, Bokhour B, Parker VA, et al. Exploring explanatory models of risk in breast cancer risk counseling discussions: NSABP/NRG Oncology Decision-Making Project 1. Cancer Nurs 2019;42:3-11.

29. Harzheim L, Lorke M, Woopen C, Jünger S. Health literacy as communicative action-A qualitative study among persons at risk in the context of predictive and preventive medicine. IJERPH 2020;17:1718.

30. Habermas J. Theorie des kommunikativen Handelns. 1st ed. Suhrkamp, 1981.

31. Sanders T, Campbell R, Donovan J, Sharp D. Narrative accounts of hereditary risk: knowledge about family history, lay theories of disease, and "internal" and "external" causation. Qual Health Res 2007;17:510-20.

32. Hallowell N. Balancing autonomy and responsibility: the ethics of generating and disclosing genetic information. J Med Ethics 2003;29:74-9.

33. Maddock C, Camporesi S, Lewis I, Ahmad K, Sullivan R. Online information as a decision making aid for cancer patients: recommendations from the Eurocancercoms project. Eur J Cancer 2012;48:1055-9.

34. Farmanova E, Bonneville L, Bouchard L. Organizational health literacy: review of theories, frameworks, guides, and implementation issues. Inquiry 2018;55.

35. Holmberg C, Bandos H, Fagerlin A, et al. NRG Oncology/National Surgical Adjuvant Breast and Bowel Project Decision-Making Project-1 Results: decision making in breast cancer risk reduction. Cancer Prev Res (Phila) 2017;10:625-34.

36. Dixon-Woods M, Williams SJ, Jackson CJ, Akkad A, Kenyon S, Habiba M. Why do women consent to surgery, even when they do not want to? An interactionist and Bourdieusian analysis. Soc Sci Med 2006;62:2742-53.

37. Sarangi S, Bennert K, Howell L, Clarke A. 'Relatively 
speaking': relativisation of genetic risk in counselling for predictive testing. Health Risk Society 2003;5:155-70.

38. Bloom DL, Chapman BM, Wheeler SB, et al. Reframing the conversation about contralateral prophylactic mastectomy: Preparing women for postsurgical realities. Psycho-Oncology 2019;28:394-400.
39. Holmberg C, Waters EA, Whitehouse K, Daly M, McCaskill-Stevens W. My lived Experiences are more important than your probabilities: the role of individualized risk estimates for decision making about participation in the Study of Tamoxifen and Raloxifene (STAR). Med Decis Making 2015;35:1010-22. 